\title{
Situations critiques étendues : la singularité physique du vivant ${ }^{1}$
}

\author{
Francis Bailly \\ Physique, CNRS, Meudon \\ bailly@cnrs-bellevue.fr
}

\author{
Giuseppe Longo \\ LIENS, CNRS - ENS, Paris \\ http://www.di.ens.fr/users/longo
}

\section{Introduction}

Les mathématiques ont un rôle normatif en physique (elles ont organisé le réel, en particulier depuis le calcul infinitésimal et, plus tard, par la géométrisation de la physique); elles méritent d'avoir un rôle similaire, si possible, dans les autres disciplines. L'idée commune selon laquelle les mêmes outils mathématiques qui ont eu tant de succès en physique paraissent jouer un rôle similaire en biologie est souvent basée sur la confusion entre la dépendance des phénomènes biologiques vis-à-vis des phénomènes physiques, et leur réductibilité. Le fait que les phénomènes de vie dépendent de la structure physique est une présupposition de base pour n'importe quel moniste ; la réduction à la physique est une opération théorique : on réduit le vivant à une organisation conceptuelle donnée et propre à l'inerte. Cela étant, la première est la présupposition de la science moderne, la seconde doit être implémentée, et c'est en général le cas lorsqu'on fait référence aux théories physiques existantes, qui peuvent être inadéquates pour remplir cette tache.

Dans cet article, nous allons nous essayerons de développer des concepts organisateurs de quelques phénomènes biologiques, dans le dessein d'unifier des faits différents. C'est un essai très modeste de "unifier par concepts", une tentative qui devrait toujours précéder la mathématisation. Malheureusement, la pression du temps oblige souvent à faire des calculs avant de comprendre et de conceptualiser. Cependant, les mathématiques elles-mêmes sont le résultat d'une conceptualisation progressive, où les notions cruciales et les structures sont le résultat d'une stabilisation difficile d'exercices informels. Dans ce sens, les invariances conceptuelles parfaitement stables des mathématiques sont le fruit d'une "praxis », d'une tentative active de comprendre et d'organiser le monde. La géométrie grecque, disons, n'est pas sortie d'une approche axiomatique, mais a été le résultat d'un longue pratique d'abstraction à partir de l'action et la mesure dans l'espace physique et sensible. L'utilisation de l'infini en acte par Newton et Leibniz, dans le calcul des mouvements finis, vitesse et accélération, est dans la continuation de plusieurs siècles de débats sur la différence entre l'infini potentiel et l'infini en acte, souvent même de nature religieuse. Ensuite, l'unification des physiques sub-lunaire et supra-lunaire, la contribution majeure de Newton puisqu'elles étaient considérées jusque là comme relevant d'ontologies bien distinctes, a été obtenue par des mathématiques radicalement nouvelles, à l'origine du calcul infinitésimal moderne. En général, l'unification de niveaux objectifs (ou épistémiques), différents (en apparence), nécessite vraisemblablement de nouveaux outils techniques ou même de nouvelles organisations conceptuelles de l'objet; elle est le résultat d'une nouvelle synthèse, pas seulement d'un transfert de techniques (mathématiques) - même si une bonne pratique de ce transfert peut éventuellement aider à trouver des nouveaux outils. Dans ce dessein, nous

\footnotetext{
${ }^{1}$ Conférence invitée aux Actes du Colloque "Détermination et complexité", Cérisy, Juin 2004, (P. Bourgine, ed.), La Découverte, Paris, 2006. Certains thèmes présentés ici développent des idées en partie esquissées dans [Bailly, Longo, 2003].
} 
essayons ici d'unifier, par des concepts relativement techniques, quelques phénomènes et structures biologiques, et ne faisons que suggérer une possible mathématisation. Dans cette tentative trans-disciplinaire, nous prévoyons d'appliquer notre expérience de «théorisation » à la biologie, laquelle diffère de la pratique biologique, mais qui pourtant s'applique sur la base d'un dialogue rigoureux avec des praticiens de cette discipline.

Un point de vue de moniste, comme le nôtre, ne devrait pas nous rendre aveugle : il n'y a aucun doute sur le fait que la "matière physique » est unique et qu'il n'y a rien d'autre audelà de cela dans le monde. Pourtant les méthodes que l'on s'est données afin de comprendre les phénomènes naturels tout au long de l'histoire ne sont pas unifiées et nous ne pouvons pas prétendre les unifier par un monisme méthodologique forcé. Même la physique, en tant que construction théorique, est loin d'être unifiée (par exemple la Mécanique Quantique et la Théorie de la Relativité Générale). En bref, nous sommes monistes pour la matière, non pour la méthode, puisque l'unité est dans l'objet et non nécessairement dans les théories existantes, au sein desquelles l'unité doit être construite. Le dialogue des disciplines pourrait nous mener à de nouvelles idées et synthèses méthodologiques.

\section{Sur les singularités et la criticité en général.}

En physique, un état critique (lié à un changement de phase et à l'apparition de comportements critiques de certaines grandeurs d'état du système - aimantation, densité, par exemple - ou de certaines de ses caractéristiques particulières - comme la longueur de corrélation) est susceptible d'apparaître à l'équilibre (flux nuls) ou hors d'équilibre (flux non nuls). Si, dans le premier cas, le traitement mathématico-physique est satisfaisant (thermodynamique pour l'aspect macroscopique, renormalisation pour l'aspect microscopique), en revanche, dans le second, on est loin de disposer de théories aussi satisfaisantes dès lors que l'on est loin de l'équilibre. Il est difficile de rassembler sous une seule caractérisation les phénomènes de type critique, mais une signature commune est donnée par la divergence des longueurs et temps de corrélation ; des cas typiques sont donnés par les transitions de phases. Un autre aspect mathématique commun est donné par le fait que l'ensemble des points critiques forme un ensemble de mesure nulle (un ensemble discret de points) relativement à l'évolution du (ou des) paramètre (température, par exemple). On remarque que la situation critique, dans le cas à flux nul, apparaît comme a-temporelle, tandis que loin de l'équilibre, du fait des flux (et bien que l'on demeure à l'état stationnaire pour le système) il existe une échelle de temps naturelle.

\subsection{Du gaz au cristal.}

Un cristal et un gaz sont à l'opposé de l'échelle, en ce qui concerne l'ordre. Pour l'analyse du passage d'un état à un autre, la transition de phase, comme le passage de gaz au liquide, puis au cristal, la physique suggère deux éléments analytiques concernant ces changements d'états : les notions de transition de phase et de longueur de corrélation.

Ces notions concernent le passage par un état critique, qui change radicalement les propriétés et les paramètres. En particulier, les transitions de phases changent la longueur de corrélation, qui, dans certains cas, peut-être interprétée comme l'établissement d'une "structure de cohérence" (dans certains cas : comme la distance des relations causales) entre les éléments de la structure physique. Lors du changement d'état, la structure globale est entièrement impliquée dans les activités de la particule : la situation locale dépend du (est corrélée au) global. Mathématiquement, cela pourrait être exprimé par le fait que la longueur de corrélation tend formellement vers l'infini ; physiquement, cela veut dire que la détermination est globale et non plus locale. Cependant, la transition survient souvent à une longueur finie. En tout état de cause, le traitement le plus précis fait usuellement recours à la théorie de la 
renormalisation des mesures et des paramètres au point de transition ${ }^{2}$. Evidement, en physique, un état critique se réduit le plus généralement au point critique et la renormalisation, qui permet de rendre compte du passage du local au global, se trouve cantonnée à la valeur du paramètre pour laquelle a lieu cette transition, ce qui finalement ne fait dépendre implicitement cette renormalisation que d'une seule valeur de ce paramètre (en laissant de côté ici la dépendance en fonction de la dimension de l'espace de plongement ou de celle du paramètre d'ordre).

\subsection{Du local au global.}

Lors des transitions critiques (changements de phase), plusieurs caractéristiques manifestent le passage du local au global relativement à la pertinence des objets décrits et de leur interaction : divergence de la longueur de corrélation (portée des interactions), apparition d'un ordre (mesuré par un paramètre d'ordre qui devient non nul, brisure de symétrie), apparition d'exposants critiques associés à des discontinuités ou des divergences (susceptibilités), apparition d'une non-analycité de l'énergie libre, du point de vue thermodynamique, .... .

Dans le cas mentionné plus haut de la transition critique du liquide au cristal, par exemple, un ordre ou réseau apparaît, celui de la structure cristalline. Un autre exemple classique est celui de la transition paramagnétique/ferromagnétique dans un système de spin. Lorsque l'on s'approche de la température critique (température de Curie) le moment magnétique global (qui sert ici de paramètre d'ordre) devient non nul (phénoménologiquement, cela revient à considérer que la composante entropique de l'énergie libre, dominante à haute température à cause du désordre thermique ainsi engendré, devient secondaire devant la composante proprement énergétique due à l'interaction entre spins, dont l'alignement diminue cette énergie, tout en étant à l'origine de l'apparition du moment magnétique global). En même temps, les corrélations (dont l'effet à la distance $\mathrm{r}$ d'une perturbation est généralement en exp($\mathrm{r} / \mathrm{L}$ ), où $\mathrm{L}$ est la longueur de corrélation) acquièrent une portée de plus en plus grande jusqu'à intéresser le volume total du système, du fait que la longueur de corrélation L diverge en $(\mathrm{T} / \mathrm{Tc}-1)^{-v}$ (Tc est la température critique de transition et $v$ un exposant critique (souvent égal à 1/2)). De même, la susceptibilité magnétique diverge avec son propre exposant critique (ce que l'on peut commenter en disant qu'au point critique à une cause finie répond un effet infini, ou qu'à une cause infinitésimale répond un effet fini).

Ces exposants critiques eux-mêmes peuvent être calculés au moyen de la technique du groupe de renormalisation (rendue possible du fait de la longueur de corrélation infinie qui rend le système invariant d'échelle au point critique). Il résulte de ces caractéristiques que "l'objet pertinent" (pour l'observation et la théorie) change au point critique : de local (les spins individuels désordonnés et corrélés uniquement aux plus proches) l'échelle intéressante devient globale (le moment magnétique du système pris comme un tout, du fait de la grande portée des corrélations).

Avec ces exemples simples, nous avons vu à l'oeuvre les notions d'état critique, de transition de phase, de longueur de corrélation (voir [Lesne, 2003] et ses nombreuses exemples et références pour en savoir plus). L'échelle d'observation importe de façon cruciale : un point

\footnotetext{
${ }^{2}$ La renormalisation en une technique mathématique originaire, dans ses méthodes modernes, de l'électrodynamique quantique. D'un point de vue conceptuel, c'est une redéfinition de l'objet considéré, en ajoutant à ses caractéristiques initiales (typiquement sa masse) certaines classes d'interactions qui les modifient. Plus généralement, le "groupe de renormalisation" fournit l'outil mathématique utilisé pour représenter le passage du local au global le long des transitions critiques, dans maints domaines de la physique : la renormalisation décrit alors un changement de mesure et d'objet, obtenu en intégrant les nouvelles classes d'interaction dues à la transition.
} 
critique comme maximum pour la complexité moyenne pourrait être vu comme un point de divergence comme la longueur de corrélation, à une moindre échelle.

En conclusion, aller du local au global, en physique, nécessite le passage par un état critique ; cela est compris comme une divergence mathématique de la longueur d'interaction, donc comme un point où les variations infinitésimales créent des changements finis (ou des variations finies menant à des changements infinis, mathématiquement).

D'un point de vue conceptuel, du fait de la détermination essentiellement globale d'un objet biologique par rapport à ses composants locaux, on est tenté de faire le rapprochement avec ces situations critiques, mais en les considérant cette fois spatio-temporellement étendues, et de considérer certaines limites infinies (d'états physico-chimiques) comme co-constituantes de cet objet biologique. De ce point de vue, l'existence et le maintien du vivant serait alors assimilables à l'existence et au maintien d'un situation (ou zone) de criticité étendu(e). Sous l'angle de la complexité, il en résulterait que le vivant, quel que soit le niveau auquel on le considère, présenterait une complexité infinie par rapport au physico-chimique, sans pour autant renoncer à hiérarchiser ces niveaux eux-mêmes selon des ordres d'infinité variés (ou de renormalisations successives). Dans la section suivante, on essayera de développer ce point de vue.

\subsection{Transitions de phase dans la criticité auto-organisée et "order for free".}

Le rôle des transitions critiques pour l'analyse du vivant a été déjà mis en évidence par d'autres auteurs. L'idée au coeur des approches développées essentiellement à partir de l'article de 1988 par Bak, Tang et Wiesenfeld est que la physique propose maints exemples de construction de "self-organization" près des états critiques, loin de l'équilibre, une idée déjà au cœur de [Nicolis, Prigogine, 1977]. Un autre regard au sujet de la "self-organized criticality" voit celle-ci émerger du chaos: "order for free", dans la terminologie de [Kauffman, 1995] (voir aussi [Solé, Goodwin, 2000]). Tout en reprenant rapidement le point de vue de [Kauffman, 1995], la simple interaction d'éléments a priori non-organisés, peut être à l'origine de la formation de structures organisées. C'est le réseau même qui corrèle des éléments simples qui produit l'émergence (un mot clé dans ces approches) de structures ou phénomènes complexes. And this, possibly by reaching "for free" a critical point: the early and paradigmatic case, analyzed in [Bak et al., 1988], is provided by the sand piles (dropping sand in a point up to the formation of a pile and, then, avalanches).

Ce que les analyses que nous développerons ci-dessous partagent avec ces approches, surtout celle proposée par Kaufman, est le rôle attribué à l'émergence de structures de cohérence au cours des "transitions de phases", en tant que passage d'un état, voire d'un processus, à un autre. "Life may exist near a phase transition", propose explicitement Kauffman, [Kauffman, 1995] : elle paraît située entre ordre et chaos, "on the edge of chaos", car trop d'ordre, voire l'équilibre, correspond à la mort, tandis que le chaos est l'opposé de l'organisation (et le vivant est très organisé). Dans les cas étudiés, la notion de "edge of chaos", un point de passage entre ordre et désordre, a une signification physico-mathématique bien précise et elle fait toujours référence à un point mathématique dans l'espace des paramètres (ou, le plus souvent, $d u$ paramètre) de contrôle.

Ce qui intéresse particulièrement dans ces transitions, critiques dans le sens mathématique mentionné, est que la structure globale, qui se constitue (émerge) dans la transition, est entièrement impliquée dans les activités locales et vice-versa (l'aimant qui se forme à la température critique, de Curie, corrèle les spins locaux et l'orientation globale). Bref, la situation locale dépend du global, par une "structure de cohérence", émergente au paramètre critique. Mathématiquement, cela est exprimé par le fait que la longueur de corrélation tend formellement vers l'infini ou que des variations infinitésimales du paramètre de contrôle 
induisent des changements finis dans les observables; physiquement, cela veut dire que la détermination est globale et non plus locale.

L'idée donc d'utiliser la physique de la criticité, avec ses "formations de structures de cohérence" pour analyser l'émergence de la stabilité structurelle du vivant, est aussi au cœur de nos analyses ; car cette physique de la criticité développe depuis une vingtaine d'année une belle théorie de l'émergence de formes organisées en présence de transitions critiques. Ces structures se "constituent toutes seules" (order for free): la criticité s'auto-organise, donc.

Voici quelques caractéristiques de cette auto-organisation, vue par les auteurs mentionnés:

- l'existence de nombreux états stables

- le passage de l'un à l'autre de ces états peut dépendre de fluctuations (infinitésimales)

- la création d'une structure spatio-temporelle dans un milieu à l'origine homogène;

- l'existence de bifurcations, qui se présentent à des valeurs bien précises des paramètres de contrôle (valeurs critiques) ; en franchissant ces valeurs exactes, et seulement dans ces cas, ces structures de cohérence apparaissent.

Dans le cas paradigmatique, la formation du tas de sable, quand un certain angle critique est atteint, par l'ajout de grains de sable, des avalanches se forment, selon une structuration mathématique bien précise. Une des limites de cet exemple, au coeur des analyses de Per Bak, est que l'auto-organisation se réduit à atteindre spontanément (for free) un angle critique et osciller autour de cet angle. Moreover, these dynamic sand piles are a dissipativemorphogenetic processes, but they are not inherently dissipative: the sand pile stabilizes if the flux of energy/matter stops; particularly, this distinguishes them from the autopoietic and homeorhetic phenomena essential to life. Nor there seem to be a global coherence structure neither a global correlation length : the arrival of a new sand grain produces avalanches at all scales, a very interesting phenomenon, but insufficient to develop the views on life we will focus on (cell activities do not seem to be scale invariant).

Par contre, un élément d'intérêt que l'on trouve dans d'autres exemples plus complexes, c'est que les règles de formation peuvent changer au cours du temps et selon les caractéristiques du système : on est en présence d'une "dynamique adaptive" [Solé, Goodwin, 2000].

En général, la physique de la criticité permet de traiter maints cas où ce sont moins les individus que les interactions qui comptent. Or, il ne fait pas de doute que la biologie (et la cognition) sont avant tout une histoire d'interactions, de formations de structures organisées (cohérentes), relativement stables. En plus, des seuils critiques apparaissent partout : des mutations génétiques, qui se propagent seulement à partir d'un certain seuil critiqueprobabiliste, aux potentiels d'action des neurones. De même, on peut analyser nombreuses activités cérébrales en termes d'attracteurs (des structures cohérentes qui apparaissent au-delà "of the edge of chaos", dans des situations proprement chaotiques), où ces attracteurs se forment et disparaissent dynamiquement, dans un milieu excitable. La moindre perturbation peut engendrer le passage d'un seuil critique, typiquement "at the edge of chaos", qui déclenche des cascades d'activités différentes : la théorie de la criticité peut donc nous renseigner aussi sur ce sujet. Est-ce que les méthodes de la physique suffisent à rendre ces aspects du vivant intelligibles?

Nous le croyons, en partie, car il s'agit d'une piste nouvelle et intéressante pour les analyses de la complexité du vivant et de sa "singularité physique". Le cadre reste toutefois entièrement interne à la physique et pas un seul des exemples dans la littérature ne demande des outils différents de ceux qui sont utilisés pour des structures inertes (à l'exception peutêtre des "correlated landscapes" de Kauffman, sur lesquels on reviendra). En fait, la plupart des exemples suffisamment mathématisés sont de nature physique ou ne représentent que la "friction physique" du vivant à son intérieur ou à son environnement: une composante essentielle de son être au monde, mais fort incomplète. Essayons de nous expliquer par un 
exemple. Les structures fractales de maints organes (poumon, systèmes vasculaires...) sont le résultat d'un problème d'optimalité (maximiser l'échange énergétique par une surface, dans un volume...) où la structure physique se forme en présence d'un seuil critique. La "poussée" (croissance) du vivant est essentielle pour cela, mais l'interaction est physique : on peut reproduire des structures optimale similaire avec des matériaux inertes (la cire des abeilles, la fractalité présente dans maintes structures physiques ou de simulation informatiques...). Ces structures sont des "géodésiques" dans l'espace de phases (paramètres et observables) approprié, comme pour toute évolution physique ; une de leurs caractéristiques principales réside dans la ponctualité des passages critiques (les valeurs critiques des paramètres sont des points mathématiques). Toute la phyllotaxie, aux mathématiques fort développées et intéressante, représente cette friction entre la poussée du vivant et le milieu physique ; elle décrit toujours, et ce n'est pas un hasard, des phénomènes au niveau des organes, (voir [Bailly, Longo, 2003] : lieu d'échange, énergétique en particulier, entre organisme vivant et milieu physique). Encore une fois, l'utilisation faite de la physique de la criticité par Bak, Kauffman ... est d'un très grand intérêt. Elle donne un aperçu possible, en particulier, de l'émergence de la vie à partir de l'inerte [Kauffman, 1995]. Elle enrichie l'analyse de l'évolution darwinienne, ancrée sur la sélection, par un moteur qui lui manquait: la construction de l'ordre à partir du désordre, sur la base de seules considérations physiques ; citons, par exemple, les réseaux (métaboliques, informationnels...) qui se stabilisent au-delà d'un certain seuil, voire l'émergence de corrélations structurellement stables.... En fait (et les démonstrations en sont désormais nombreuses), le hasard des mutations ne suffit pas pour comprendre la phylogenèse : la formation spontanée de l'ordre, "order for free", fournit une alternative fascinante (et pertinente) - un ordre ensuite soumis à la sélection, bien évidemment.

Ces analyses, toutefois, s'arrêtent au "seuil de la physique": elles nous amènent "at the edge of chaos", qui reste une transition ponctuelle, comme partout en physique (une valeur bien précise des paramètres de contrôle). Une ponctualité bien nécessaire aux méthodes physico-mathématiques largement utilisés dans ce domaine : le groupe de renormalization. (Un des plus beaux exemples à cet égard, la percolation, [Solé, Goodwin, 2000 ; Lesne, 2003], est intéressant justement et surtout pour la ponctualité de la transition critique ; mais cette ponctualité est tout à fait générale, voir [Solé, Godwin, 2000]). Le nombre modeste de paramètres, voire un seul, par rapport auxquels on examine la criticité, est aussi un aspect essentiel (parfois souligné comme positif) de ses approches et des méthodes de renormalisation. La formation de la vie elle même serait alors une transition critique, par rapport un ou deux paramètres; et la vie se situerait, avec ses phénomènes propres, dans une zone proche, mais au-delà, du seul critique.

Or, de notre point de vue, la singularité physique du vivant consiste, entre autres, dans la robustesse de la criticité, qui tolère une extension du passage critique. Le "edge of chaos" ponctuel des analyses physiques ne nous paraît plus suffisant : il doit se transformer en un intervalle par rapport à tous les paramètres pertinents (très nombreux: espace-temps, température, pression...). Dans un certain sens, les travaux cités essayent de rendre intelligible la formation de la vie, à partir de l'inerte, en tant que passage critique, mais ils n'examinent pas proprement la vie perdurante, sa "criticité étendue". Notons également que la singularité conceptuelle du vivant se situe aussi dans la dynamique de l'espace de phase, nous y reviendrons.

Pour nous résumer, le défi mathématique, par rapport aux théories physico-mathématiques courantes, consiste dans la non-ponctualité de la stabilité structurelle du vivant (criticité étendue par rapport à nombreux paramètres de contrôle) ainsi que dans la difficulté à établir un paysage (espace de phase) figé, à l'intérieur duquel tout processus se déroulerait, suivant des géodésiques ponctuées par des transitions critiques. À cet égard, c'est l'espace de phases 
lui-même qui change dynamiquement (un nouvel organe, une espèce - des observables et des paramètres inattendus - poussent au cours de l'ontogenèse, de la phylogenèse) : la dynamique est aussi dans les observables et les paramètres mêmes de l'écosystème, un cadre co-évolutif où l'émergence de la nouveauté change les donnes de bases. La notion de Kauffman de "correlated landscapes", avec leurs dynamiques, et les "dynamiques adaptives" [Solé, Goodwin, 2000] sont proches de l'idée que nous mentionnons dans ce texte ; elles sont même très riches, du point de vue mathématique en particulier, car la première, par exemple, spécifie une notion fort intéressante de "corrélation" dans un espace de phase normalement considéré comme régi par le hasard. Elles paraissent toutefois forcer une stabilité conceptuelle et mathématique, propres aux théories physiques (l'espace des paramètres et des observables est donné a priori) qui est insuffisante, à notre avis, pour cerner les dynamiques évolutives du vivant (voir plus bas).

Par contre, dans ces espaces évolutifs changeants, ce qui est relativement stable et robuste c'est la criticité perdurante de l'objet vivant : autant qu'il reste dans une plage des possibles (en s'adaptant), l'individu vivant survit sur un parcours générique (possible, et pas nécessairement optimal) dans des espaces (des écosystèmes) intrinsèquement changeants. Et cela également en vertu de l'extension de sa situation critique. Notre esquisse conceptuelle se situe donc à la limite des théories physiques de la criticité et essaye de saisir certains aspects de la phénoménalité propre au vivant, dans l'espoir qu'une unité (ou... une transition critique) puisse ensuite s'établir avec les concepts et, surtout, les mathématiques, de l'émergence et de l'auto-organisation physique. We finally observe that the notion of extended criticality, as developped here, was first presented in [Bailly, 1991b], whose scientific reference goes back to the early work on self-organised non-equilibrium systems, summarized in [Nicolis, Prigogine, 1977], more than to the approach by the authors above (see also the end of the next section, where we better stress the difference).

\section{La vie en tant que "situation critique étendue".}

Nous avons vu qu'un état critique, en physique, est une singularité dans un processus : c'est-àdire, tout au long du processus, une position, une configuration, un "état » qui peut être accessible brièvement avant que l'état global de ce que l'on observe ne change (radicalement). Un état critique peut aussi être vu comme une bifurcation ou bien, dans certains cas, comme une catastrophe, dans le sens de Thom, en particulier si ce changement devient irréversible. Dans un sens, c'est l'opposé d'une situation d'équilibre (le signe opposé dans la description mathématique); et elle diffère aussi de la notion d' "être loin de l'équilibre », puisque cette situation, en général, n'implique pas d' "évolutions différentes possibles » du système (bifurcations).

Par définition, un système ne peut pas rester «longtemps» dans un état critique : le développement au cours du temps le force au-delà de cet état. C'est un aspect typique de transition, où des fluctuations mineures, possiblement en dessous de niveau d'observabilité, peuvent mener à des évolutions radicalement différentes. La nature instantanée du critique est merveilleusement bien exprimée en mathématiques par la divergence (vers l'infini, voir précédemment) des descriptions fonctionnelles, selon ses paramètres propres. Ou, aussi, comme nous l'avons discuté précédemment, par le maximum de complexité, qui lui aussi crée l'instabilité.

Par contraste avec les situations physiques, il semble bien que le vivant (que l'on peut représenter, au moins sous une certaine durée, comme un état stationnaire loin de l'équilibre) évolue dans une "zone critique étendue", qui dure dans le temps et dont la criticité pourrait être représentée par un ensemble dense, voire continu, de points critiques dans l'espace de phase. Ce trait serait rendu possible la fois par la clôture organisationnelle spatiale d'un 
organisme, par sa finitude dans le temps et par son autonomie homéostasique (intégration, régulation). D'autre part, comme nous l'avons déjà souligné ailleurs, ce vivant se caractérise par la coexistence et l'articulation de plusieurs types de temporalité (cycles pour les horloges internes, relaxations pour les phénomènes de stimulus/réponse, internes ou externes). Il lui correspond aussi une hiérarchie enchevêtrée de niveaux d'organisation de natures différentes (biolons et orgons ${ }^{3}$ ) et le passage d'un niveau à un autre n'est plus du tout comparable à celui qui s'opère en physique pour le système étudié, au moyen de la renormalisation par exemple, entre le local et le global en un point critique donné : il existe désormais des caractéristiques intrinsèquement différentes au sein même du système selon les niveaux et échelles, ce que manifeste l'introduction de concepts étrangers à ceux de la physique, tels, avons nous vu, que ceux d'intégration et de régulation, couplés à celui de fonction biologique d'une part, de finalité contingente ou d'anticipation, d'autre part (ce qui modifie en même temps le régime de causalité du vivant par rapport à celui de la physique $)^{4}$.

Notre thèse, donc, est que la notion de criticité physique pourrait nous aider à comprendre un état biologique comme une singularité physique «de longue durée», une "situation critique étendue», dans lequel, en particulier, les processus homéorhésiques maintiennent une tension permanente entre local et global. Une situation physique qui, mathématiquement, est comprise comme le lieu d'une divergence, un passage du fini à l'infini, et comme le point singulier où l'objet pertinent change (renormalisation, voir note 2) : le local est intégré en un nouvel objet, l'objet global (l'unité vivante ou biolon). Ainsi, en termes physiques, la mesure de la complexité objective de la moindre unité vivante, une cellule, une structure globale et ses composants, a une valeur infinie, dans un sens mathématique précis. De même, si l'on considère des paramètres physiques comme pour des mesures (la longueur de corrélation et ses effets relatifs), l'élément vivant est dans un état critique progressif, un passage permanent entre local et global. C'est ainsi qu'une unité dynamique singulière, celle du vivant, à partir de la cellule, est infiniment plus complexe que n'importe que processus physique, qui peut se comporter de façon critique seulement dans des cas exceptionnels, de courte durée, des singularités.

Pour nous résumer, une unité du vivant, un biolon selon notre terminologie, instable de façon critique, est conservé dans sa situation étendue, loin de l'équilibre statique, par homéostasie, ou mieux, par homéorhésie. Ou bien, l'intégration dynamique et la régulation de ses composants (les orgons, avec leurs composants, les biolons, avec leurs orgons...), leurs relations «ago-antagonistes" [Bernard-Weil, 2002] à l'intérieur d'eux-mêmes et de l'environnement, les soutiennent dans une situation physique improbable. L'autopoièse constitue une autre manière d'exprimer cette dynamique auto-constitutive, [Varela, 1989; Bourgine, Stewart, 2004]. Une organisation mathématique qui peut être liée à l'autopoièse pourrait faire référence à plusieurs endomorphismes couplés, en termes mathématiques ; leur

\footnotetext{
${ }^{3}$ De façon synthétique, nous appellons une entité vivante biolon (une cellule, un individu, animal ou végétal, une espèce entière). Les biolons sont composés d'orgons (les organelles d'une cellule, les organes d'un individu, les populations organisées d'une espèce). Cette unification de terminologie est justifiée par l'uniformité des concepts, voire des outils mathématiques, avec lesquels on peut traiter les trois niveaux rassemblés sous le même nom (voir [Bailly et al., 1993] et [Bailly, Longo, 2003]).

${ }^{4}$ Une image permet peut-être de mieux illustrer ce que l'on peut entendre par là : si on représente la causalité efficiente de la physique comme une flèche allant d'un point initial à un point final, sur une ligne, la ligne en question serait infinie et formerait ainsi un support non compact ; la causalité biologique, qui s'ajouterait à la causalité physique, pourrait alors être représentée par une même flèche mais sur une ligne fermée, cette fois, formant un support compact, représentant les effets de rétroaction et de finalisation (associés peut-être aussi aux effets de clôture - et d'autonomie - évoqués par ailleurs). Passant à un autre niveau d'analyse, on pourrait considérer que ce type de manifestation causale se manifeste sur des fibres internes constituant des dimensions supplémentaires compacifiées. Les deux structures causales sont bien évidemment compatibles et "simultanées", car le vivant est immergé dans les champs physiques.
} 
"clôture organisationnelle" peut alors correspondre aux limites des attracteurs structurellement stables; l'intérieur et les membranes peuvent être comprises respectivement comme les bassins et les bords des attracteurs.

Dès que l'intégration ou la régulation ne fonctionne plus ou dépasse les limites de l'état non pathologique, tout s'effondre : l'entropie grandit subitement, le désordre représente la mort. Courir sur une corde tendue est une bonne image de l'état progressif d'un biolon : lorsque le contrôle, en tant que régulation et intégration, décroît jusqu'à un certain niveau (les bords critiques de la situation critique étendue, comme limites acceptables de la pathologie) la mort termine cette vie contingente, par un état de transition final et irréversible. Bien sûr, aux bords de la situation critique étendue, transitions de phases, changements de la longueur de corrélation, passage par des singularités... surviennent continuellement, mais, à l'intérieur des limites considérées, ils sont confrontés à une activité régulatrice. En fait, ils en sont une partie essentielle : tous les seuils biochimiques qui contribuent aux échanges internes d'un biolon, peuvent être perçus comme des composants élémentaires de l'homéorhésie globale. Plus globalement, la vie elle-même peut être vue comme une "singularité physique étendue ».

Les paradigmes physiques nous ont aidé à formuler cette notion, qui n'est pas de nature physique. Le monisme, intrinsèque à la science moderne, est de la matière, non pas de la méthode ; en fait, nous faisons face à différentes phénoménalités et les rendons intelligibles en les organisant au moyen de notions et structures conceptuelles (et, si possible, mathématiques) différentes. Une synthèse est loin d'être évidente ; elle doit être construite par une nouvelle unité conceptuelle (et mathématique) en tant que un but à long terme.

Comme nous l'avons déjà discuté, la formation d'une telle zone critique étendue, associée aux conditions de possibilité du vivant, pourrait provenir de la convergence de deux processus d'origines opposées : d'un côté (première limite de la zone), à partir de l'équilibre, la suite de bifurcations critiques qui pourraient être engendrée par un écart de plus en plus importants aux situations d'équilibre (cf. Nicolis) et, de l'autre côté (deuxième limite de la zone) la criticité auto-organisée provenant de la stabilisation d'états originairement chaotiques ${ }^{5}$. La contrainte de coexistence de ces deux limites serait précisément associée à la formation de niveaux d'organisation interagissant entre eux, à la clôture organisationnelle de l'autopoïèse et finalement à la constitution d'une autonomie homéostasique susceptible de reproduction ${ }^{6}$.

\subsection{Situations critiques étendues, réflexions.}

Que peut signifier, pour un système, d'avoir à évoluer dans une "zone critique étendue" ?

D'abord sans doute de se situer à tout moment loin de l'équilibre du fait que le maintien de son organisation nécessite des échanges énergétiques intenses autorisant le maintien d'une entropie "anormalement" basse par rapport à la situation d'équilibre.

Ensuite, et corrélativement, de se présenter en effet avec une organisation interne, qui peut aller jusqu'à la constitution de niveaux d'organisation, correspondant aux contraintes de dissipation (structures dissipatives) dans le cadre de ces échanges.

\footnotetext{
${ }^{5}$ Cette situation critique étendue n'existe et ne peut se maintenir que loin de l'équilibre thermodynamique et en présence active d'échanges de matière, d'énergie et d'information avec l'environnement; en cela elle se rapproche des conditions dans lesquelles se constituent des structures dissipatives et se distingue en même temps des situations de criticalité auto-organisées (modélisées notamment par le comportement des tas de sable) telles qu'elles ont été étudiées en particulier dans [Bak et al., 1988]. Ces dernières ne sont pas dissipatives en permanence: le tas de sable se stabilise si le flux de matière énergie s'arête; en particulier cela le distingue des phénomènes autopoiétiques et homéorhésiques essentiels au vivant.

${ }^{6}$ On sait qu'en physique, ce sont bien les conditions aux limites qui engendrent (pour les cordes vibrantes, par exemple) les états stationnaires discrets solutions des équations de propagation. Peut-être peut-on y trouver une métaphore pour la constitution de ces niveaux d'organisation biologiques associés à la fois aux contraintes de clôtures organisationnelles en même temps qu'à celles de devoir évoluer dans une situation critique étendue bornée par les caractéristiques que nous venons d'évoquer (chaos, suites de bifurcations).
} 
Troisièmement de former un "tout" dans l'espace et dans le temps (au moins localement et momentanément), dans la mesure où ses longueurs de corrélation internes sont de la taille du système lui-même (état critique) et ses temps caractéristiques de parcours plus ou moins exhaustif de l'attracteur correspondant à son état sont bornés, définissant ainsi l'échelle de son existence (notamment à travers le maintien de son organisation interne). En cela se manifeste donc une première forme d'autonomie spatio-temporelle, couplée à cette hétéronomie fondamentale que constituent les nécessités soulignées précédemment d'échanges avec l'extérieur. En cela, aussi, le système se présente comme une singularité "étendue" dans le paysage physico-chimique habituel (appelant peut-être une théorie des catastrophes généralisées, extension de la théorie des catastrophes élémentaires).

Le fait d'évoluer dans une telle zone impose-t-il l'existence d'une clôture organisationnelle et l'établissement d'une frontière ? Un argument en ce sens serait associé à la nécessité de limiter les fuites et réguler les échanges énergétiques ; un autre, dans le cadre d'une autopoïèse, serait associé à la nécessité mettre en place des conditions aux limites permettant l'existence même du système ; un troisième pourrait être trouvé dans le fait que l'existence d'une zone critique étendue ne serait possible que sur une topologie permettant de distinguer un intérieur d'un extérieur (argument de compacité ?). Ces arguments ont quelque chose de circulaire dans la mesure où on a supposé l'existence du système en question pour définir ses conditions d'existence. Ce qui signifie sans doute que l'hypothèse qui consiste à associer le vivant à une zone de criticité étendue, si elle permet d'interpréter la permanence et le fonctionnement d'organismes, reste encore insuffisante pour en décrire ou expliquer l'origine. Ce qui se manifeste, à ce stade, notamment par le fait que cette hypothèse ne dit rien sur des capacités d'apprentissage - même si par certains côtés la recherche de stabilité structurelle peut vaguement s'en approcher -, ni surtout sur des capacités d'anticipation (voire finalité contingente) - même si la mise en place spontanée d'une échelle de temps caractérisant ses propres limites d'existence peut en être considérée comme un début de préfiguration.

Quant à l'objectivité de ces constructions, il ne s'agit pas seulement de distinguer dans le vivant lui-même des aspects de structure (anatomie, par exemple) et des aspects dynamiques (fonctions physiologiques, par exemple), mais aussi de porter des regards, soit de type structurel (en cela a-temporels), soit de type dynamique (en cela associés à la genèse et à la durée) sur le vivant en général.

Différentes approches, non plus seulement conceptuelles mais aussi plus ou moins mathématisées se sont depuis longtemps distribuées selon cette partition, sans qu'à notre connaissance leur articulation ait durablement été assurée. Parmi les plus récentes, citons d'un côté les applications de la théorie des singularités (catastrophes élémentaires) de René Thom et de l'autre l'application de la théorie thermodynamique des bifurcations par Grégoire Nicolis et Ilya Prigogine. Un de apports possibles devrait consister, pour le côté structurel, à recourir à une mathématisation dans le paysage de la théorie des catégories (elle aussi encore atemporelle) et pour le côté dynamique à développer une théorisation de la criticité étendue que nous venons d'évoquer en introduisant les couplages et échelles de temporalités

pertinentes. L'articulation des deux approches pourrait introduire la pertinence des temporalités dans l'aspect "catégories" et une généricité stable (voir ci-dessous) dans l'approche "criticité étendue".

\section{Les régimes d'intégration, de régulation et causaux}

Quelques faits supplémentaires peuvent ultérieurement corréler ainsi que mettre en évidence les différences entre les phénomènes physiques et biologiques. Les relations causales sont locales en physique ; elles peuvent être globales seulement dans le sens d'un champ qui relie les entités physiques par la propagation des interactions locales. Dans cette approche donc, la 
structure globale, et sa longueur de corrélation, est obtenue seulement par la transitivité des interactions locales. Ainsi, dans chacun des cas physiques (local/global), les mathématiques, en fixant un espace des phases, isolent un niveau unique de causalité. Dans la biologie, au contraire, la causalité locale peut différer radicalement des corrélations globales, pourtant elle ne peut pas être isolée de ces dernières : l'intégration et la régulation, typiquement, affectent de façon causale les interactions locales (des échanges biochimiques locaux peuvent être réglés par des cascades d'hormones ou de signaux neuraux de natures entièrement différentes). En d'autres termes, le régime causal global peut différer des régimes locaux, même s'ils interférent entre eux de manière permanente : ainsi, l'unité d'un biolon est donnée par une activité régulatoire-intégratrice, de nature physique/biochimique différente par rapport aux échanges entre et dans les orgons, quoique ces échanges soient régulés (et affectés) par le global. Notez que c'est le régime global qui contribue essentiellement à l'homéorhésie (autopoièse, couplages ago-antagonistes) comme entretien d'une situation critique étendue ; ses fluctuations, dans les frontières du critique, correspondent aux pathologies qu'un biolon peut vivre avec, comme par exemple des formes de mort locale (l'apoptose cellulaire dans un orgon). Naturellement, les fluctuations existent en physique (et peuvent être "tolérées" ), mais les entités physiques sous-jacentes ne changent pas au fil des fluctuations. Dans ce sens, dans les théories physiques, il n'y a rien qui ressemble à des phénomènes tels que des pathologies ou l'apoptose locale ; en cela, leurs mathématiques restent toujours à être inventées, autant que leur conceptualisation pré-formelle.

En résumé, l'intégration est la présence (vers le haut-causal) du local dans la structure globale, alors que la régulation est la structure globale qui affecte de façon causale (vers le bas) les structures locales. C'est cette interaction locale/globale qui organise "la conduite interne" et maintient la situation critique étendue, au moins au niveau phénoménal (voir également [Rosen, 1991; Stewart, 2002]). Mais à côté de ce rôle de stabilisation, ces interactions participent aussi de ce que l'on définira plus bas comme une (possible) indétermination théorique de l'évolution du vivant (phylogenèse et ontogenèse), car elles relient des niveaux d'organisation différents, chacun régit par son propre régime de causalité physique : on attribuera alors l'indétermination aussi aux "résonances" entre ces différentes structures causales.

Malgré la singularité physique du phénomène de la vie, essayons une fois de plus de définir notre compréhension en termes physiques familiers. La régulation peut jouer le rôle de conditions initiales ou aux limites sur le comportement global des solutions des systèmes d'équations (différentiels ou aux différences finies), c'est-à-dire des systèmes dynamiques décrits par ces équations. L'intégration peut être aussi comprise, par une analogie approximative, comme les corrélations des variables qui donnent une unité à un système d'équations donné ; ou, aussi, comme rôle d'organisation des singularités dans leurs solutions ; ou, de la même façon, comme l'extension analytique d'une solution localement définie. Une fois de plus, nous essayons juste d'approcher par des concepts physico-mathématiques une phénoménologie qui, jusqu'ici, dépasse largement ces descriptions, dans le sens mathématique d'une complexité infinie, défini dans $§ .1$ et 2 (la complexité d'une singularité).

\section{Les espaces de phases et les trajectoires.}

Les situations biologiques présenteraient toutefois un autre trait supplémentaire, tout à fait essentiel à nos yeux: cette extension spatio-temporelle ne touche pas seulement le comportement de "trajectoires" dans un espace de phase donné, mais peut se manifester aussi par une modification, par le vivant, de cet espace de phase lui-même (écosystème en un sens très vaste). Ces modifications concerneraient l'interaction avec le milieu et seraient induites par les mutations, l'apparition ou disparition de certains organes, voire populations etc.. La 
situation serait plutôt comparable à l'existence (métaphoriquement parlant) d'une sorte d'ensemble d'espaces de phase (celui correspondant à la réunion de tous les possibles pour un organisme donné), caractérisé seulement par quelques grands invariants structurels (nous pensons ici notamment aux nombres absolus qui caractérisent transversalement maintes formes du vivant, comme les rythmes métaboliques, par exemple). Ces grands espaces d'invariants seraient partitionnés eux-mêmes en (sous)espaces de phase (ceux correspondants aux réalisations effectives de ces possibles), les trajectoires évoluant généralement à l'intérieur de ces sous-espaces sur des attracteurs étranges mais étant susceptibles, sous certaines perturbations (mutations géniques, changement brutal d'environnement physico-chimique ou biologique), de passer d'un sous-espace à un autre, grâce aux propriétés de ces attracteurs (voir \$.5.1).

Toutefois, cet ensemble d'espace de phases serait loin d'être prédéterminé. Plus précisément, c'est à ce niveau, tout d'abord, qu'il conviendrait de situer une sorte d'indétermination biologique : c'est-à-dire, au niveau du passage d'un espace de phase (écosystème), à l'instant donné, à celui de l'instant successif ; et ce passage "contiendrait" ou exprimerait la trajectoire biologique (phylogénétique, ontogénétique). Pour nous expliquer, revenons brièvement aux analogies/différences avec la physique.

En physique classique, on se donne un espace de phase dans lequel se forment les trajectoires spécifiques, voire les géodésiques des objets pertinents (voir $\S .5$, plus bas); dans certains cas (non-linéaires, typiquement) la détermination formelle globale des évolutions (l'existence d'équations qui déterminent la dynamique) n'implique pas nécessairement la prédictibilité des trajectoires (mais le système reste déterministe). En revanche, en physique quantique, une fois donné l'espace des phases (les états quantiques dans un espace de Hilbert), on ne peut parler de trajectoire, dans le sens classique, et c'est une indétermination intrinsèque qui régit l'intelligibilité théorique des phénomènes ; en particulier, les relations causales sont remplacées, dans l'espace des phases donné, par des corrélations de probabilité. Ce cadre conceptuel et mathématique, avec son indétermination et ses analyses probabilistes, est au cœur de l'originalité théorique (et de la difficulté conceptuelle) de la mécanique quantique.

En revanche, nous pensons qu'en biologie, la difficulté théorique et conceptuelle réside principalement dans l'impossibilité de donner, en amont, une détermination formelle globale et un espace des phases qui puissent encadrer les phénomènes assez longuement dans le temps; c'est-à-dire, de décrire une détermination mathématique globale (un ensemble d'équations, typiquement) dans un espace des phases donné une fois pour toutes ou presque. En fait, s'il existe une relative stabilité structurelle elle concerne plus les objets que leurs cadres de vie et certaines de leurs composantes, voire leurs écosystèmes ou espaces d'observables et variables pertinents. Pour le dire autrement, les "bifurcations" se font au moins autant au niveau de la sélection entre des déterminations possibles, qu'à l'intérieur d'une détermination déjà établie.

Revenons sur ce point délicat, justement en termes de trajectoires. En mathématiques, les points critiques, sur une courbe (maxima et minima, par exemple), peuvent être dits spécifiques en ce que le plus souvent ils sont en nombre bien plus petit (un discret dénombrable) que le continu auquel appartiennent tous les autres points, qui peuvent alors être dits génériques.

En physique, à cet égard, dans l'espace des phases donné, l'ensemble des trajectoires "concevables" est générique, mais la trajectoire effective, définie par le principe géodésique, est spécifique (critique, stable, c'est-à-dire en l'occurrence, minimale pour l'action lagrangienne, ou dans le cas particulier de l'optique, minimale pour le chemin optique). Autrement dit, la phénoménalité physique effective est spécifique et s'encadre dans un espace de phases pertinent (une bonne partie du travail du physicien consiste justement à caractériser cet espace). C'est sans doute ce qui donne à la théorie physique une grande force 
mathématique ainsi qu'une possibilité, par abstraction, de caractériser les objets physiques par des propriétés très générales, malgré la singularité de chaque expérience particulière dont les conditions ne sont pas toujours exactement reproductibles : la trajectoire de n'importe quel objet sera spécifique et son analyse est rapportée à l'espace (abstrait et général) des phases.

Par contraste, il semble bien qu'en biologie, les cellules d'un organisme, les organismes d'une espèce, les espèces d'un environnement (c'est-à-dire, les biolons), sont concernés par des "trajectoires génériques" : toutes celles qui demeurent compatibles avec leur perduration, voire leurs transformations (mutations). Ce serait le repliement sur la spécificité de ce générique qui lui ferait perdre son caractère biologique chaque fois que l'on essaye une réduction au physique.

Dès lors, on pourrait comprendre la situation critique étendue comme l'expression de cette généricité par contraste avec la transition critique localisée (dans l'espace des paramètres et dans le temps, à cause des incessantes fluctuations) de la physique. Pour le dire autrement, il aurait une sorte de dualité entre physique et biologie : spécificité des trajectoires et "localité" de la criticité pour la physique, généricité des trajectoires et extension de la criticité pour la biologie. En même temps cela permettrait de relier "naturellement" le biologique à la variabilité considérable dont il est le siège puisque cette compatibilité générique autoriserait l'existence d'un grand nombre de "trajectoires" possibles. Les invariants ne devraient alors plus être définis dans un espace de phase donné mais sur l'ensemble des espaces de phase compatibles avec cette généricité, chaque spécificité pouvant modifier la structure locale de cet ensemble sans pour autant disparaître. C'est ce qui expliquerait peut-être que les grands invariants (approchés) que nous avons mentionnés concernent presque toujours des ensembles considérables d'organismes (mammifères, métabolismes oxygène, règne animal lui-même, etc.), car ils constitueraient certains des rares invariants par rapport a cette plasticité globale. Ces invariants persistent, dans la variabilité du vivant, et contribuent à le stabiliser ; de plus, cette variabilité biologique diffère de la variation physique, car cette dernière ne contribue normalement pas à la stabilité (au moins dans les cas non-linéaires) et se situe dans un cadre de détermination, tandis que la première peut le franchir.

En conclusion, nous pensons que, en biologie, c'est la collection même des objets pertinents et des paramètres (l'espace des phases) qui suivra l'état présent, qui est indéterminée et que cette indétermination devrait être intrinsèque à la théorie. Dans cet écosystème changeant, les trajectoires mêmes (notion que l'on garde alors qu'elle devient problématique en physique quantique) diffèrent de celles de la physique classique, car elles ne sont pas spécifiques (des géodésiques dans des espaces donnés), mais génériques (des possibles dans des espaces qui changent). Dans un sens, il y aurait donc deux niveaux d'indétermination, corrélés entre eux : celui du passage au nouvel écosystème et celui du "choix" de la trajectoire pour chaque biolon, parmi toutes les trajectoires compatibles. Au cours de la phylogenèse, typiquement, la formation d'une nouvelle espèce qui participe de l'écosystème, voire l'émergence d'un nouvel organe, modifie l'espace de l'évolution, voire de l'ontogénèse, et modifie l'ensemble des trajectoires possibles. Encore une fois, la généricité de la trajectoire ne serait pas à analyser uniquement dans un même espace des phases, mais aussi en termes de passage à un nouvel espace possible.

Pour cette raison, à notre avis, l'analyse des dynamiques du vivant ne peut pas se réduire aux termes de l'imprédictibilité déterministe classique (avec ses géodésiques, dont le choix est plus ou moins sensibles aux conditions aux contours), mais en ceux d'une indétermination intrinsèque de l'évolution du vivant, à comprendre en termes de généricité des trajectoires et de changement d'espace des phases. L'extension des situations critiques, conjointement à l'intrication des niveaux d'organisation et leurs effets de "résonance" réciproque, en proposeraient une intelligibilité de type physique, pour autant que la physique arrive à nous donner des métaphores adéquates, car, comme a su faire la mécanique quantique par rapport à 
la mécanique classique, il faudrait se donner des concepts et structures mathématiques autonomes, pour mieux saisir les "champs" du vivant et ses dynamiques ${ }^{7}$.

\section{Un autre regard sur la stabilité et la variabilité.}

Nous avons mentionné plus haut le rôle stabilisant des régulations et intégrations dans l'enchevêtrement et l'interaction des niveaux d'organisation. Notre analyse, en termes de généricité des trajectoires et de dynamiques des espaces des phases, devrait contribuer à rendre intelligible le fait que la vie phénoménale est non seulement caractérisée par la conservation de quelques invariants clés, en raison de la stabilité, mais également par la variabilité. Pour cette raison, nous avons considéré l'enchevêtrement et les interactions des niveaux d'organisation d'une part comme une des composantes de la stabilisation, et d'autre part comme faisant partie de l'indétermination biologique (en tant que résonances déstabilisant chaque niveau d'organisation). Suite à la variabilité, typiquement, les biolons diffèrent tous les uns des autres : les individus d'une espèce ne sont et ne doivent pas être identiques. Même les cellules ont une certaine forme d'"individualité". En effet, la variabilité est au cœur de l'évolution et, conjointement à l'individuation, elle est aussi à la base de l'ontogenèse. Clairement, c'est encore une autre singularité de la vie phénoménale par référence aux descriptions physiques, où des entités individuelles du "même type" sont toutes identiques et leurs descriptions peuvent être, conceptuellement et ainsi mathématiquement, parfaitement stables (la spécificité des trajectoires physiques dont nous avons parlé).

\subsection{Les biolons comme attracteurs et trajectoire individuelle}

Essayons maintenant de comprendre cette approche, décrit par des dynamiques indéterministes dans des cadres globalement stables, voire cette dialectique stabilité/instabilité, en termes différents, mais compatibles avec les précédents.

D'un côté, une espèce présente une stabilité structurelle globale, alors que ses membres peuvent montrer différentes variations dans leur nature et leur comportement. De même, à son échelle de temps, un individu est stable, alors que ses cellules évoluent et meurent, chacune selon un chemin différent. Dans l'un ou l'autre des cas, la structure globale est essentiellement (mais pas entièrement) préservée, pendant que des variations locales ont lieu. Si l'on prend une analogie physique, le biolon le plus grand (une espèce, un métazoaire) peut être décrit par la géométrie d'un attracteur, dont la dynamique est essentiellement stable aussi longtemps qu'il reste dans un même espace de phase. Mais on pourrait aussi comprendre les changements d'espace des phases comme le basculement de l'attracteur dans un autre espace, avec les caractéristiques d'indétermination dont on a parlé. La difficulté consisterait alors dans la recherche de bonnes classes d'universalité, voire des grands invariants biologiques, comme, par exemple, les nombres absolus (les horloges du vivant, etc.) qui concernent des espèces, voire des phyla entiers, et qui permettraient de parler d'un basculement du "même" attracteur.

\footnotetext{
${ }^{7}$ La théorie de la viabilité (voir [Aubin, 1991]) propose une analyse mathématique voisine, mais différente de l'approche que nous proposons : on remplace l'équation d'évolution $(\mathrm{dx} / \mathrm{dt}=\ldots)$ par l'appartenance à un ensemble d'évolutions possibles $(\mathrm{dx} / \mathrm{dt} \in$....) Toutefois, la fonction $\mathrm{x}(\mathrm{t})$ et l'ensemble qui déterminent ce passage sont donnés à l'avance et contiennent, selon notre interprétation, la liste des tous les espaces futurs possibles, avec leurs géodésiques ; $\mathrm{x}(\mathrm{t})$, en particulier, représente une "trajectoire" d'espaces de phases, au lieu d'une trajectoire dans un espace de phases. C'est là, pensons-nous, qu'il faut passer d'une trajectoire déterminée, à une indétermination intrinsèque comparable aux chemins possibles et indéterminés de la physique quantique, mais concernant l'espace des phases lui-même. De plus, comme nous l'avons dit à plusieures reprises, dans chaque espace, ce sont les trajectoires génériques qui jouent un rôle important et non pas seulement les géodésiques.
} 
Cette stabilité dynamique est aussi compatible avec les variations et même l'instabilité des trajectoires individuelles, qui peuvent tomber à l'intérieur du même attracteur (les biolons plus petits, inclus dans le plus grand - un métazoaire, une cellule, respectivement). Alors un biolon du niveau intermédiaire (un métazoaire) constitue une trajectoire pour une espèce, cette dernière étant considéré comme un attracteur ; en même temps, ce biolon se comporte comme un attracteur stabilisant pour ses propres composantes, ses cellules.

Comme nous l'avons observé, en physique, la dynamique de la trajectoire est donnée par la géodésique dans un espace des phases prédéfini ; ainsi, celle-ci est sélectionnée selon la stabilité la plus élevée et généralement d'une manière unique (elle est spécifique). Par contraste, les biolons individuels sont selon nous représentés par des chemins simultanés différents, génériques et soumis simplement à des contraintes de compatibilité avec les conditions aux limites (qui à leur tour n'ont pas besoin d'être stables et peuvent être modifiées par les processus continus). Cette approche permettrait de capturer simultanément les propriétés associées à :

- l'interaction de la stabilité relative globale et de la variabilité individuelle, en tant que attracteur vs. trajectoire ;

- la compatibilité au lieu de l'"optimalité", car les trajectoires doivent seulement rester dans les frontières d'évolution de l'attracteur.

Chaque trajectoire individuelle trouverait donc son origine sur un état instable et actualiserait différentes potentialités contextuelles (typiquement la première mitose dans l'embryogenèse, dans le même génotype) et évoluerait selon les potentialités accessibles, dans le cadre de la stabilité structurale globale.

La sélection naturelle peut réduire la variabilité au niveau des espèces, tout en l'augmentant au niveau des individus. Les pathologies plus ou moins supportables concerneraient plutôt les cellules chez des individus. Naturellement, ce modèle conceptuel s'adapte parfaitement à la notion proposée ci-dessus de situation critique étendue, comme état paradigmatique des biolons. D'ailleurs, il peut également être vue comme une généralisation de la notion de chréode de Waddington ([Waddington, 1977]), par les aspects de généricité et indétermination sur lesquels nous avons insisté.

Observons que ce schéma se réfère seulement aux biolons, à divers niveaux, puisque seuls les biolons sont concernés par les identités (identités préservées par les changements). Les orgons constituent plutôt, par des échanges énergétiques et des activités fonctionnelles, le support matériel à la stabilité et à la variation : un orgon n'est pas une trajectoire, mais il peut être à l'origine de la variation continue de l'individu représenté par la trajectoire.

Les paradigmes physiques nous ont aidé à formuler ces notions, qui ne sont pas proprement de nature physique. Nous rappelons, encore une fois, que, tout en travaillant dans un cadre moniste, nous faisons face à différentes phénoménalités. On les rend intelligibles en les organisant au moyen de structures conceptuelles (et, si possible, mathématiques) différentes, avant de viser une "unification". Une synthèse, en fait, est loin d'être évidente ; elle doit être construite par une nouvelle unité conceptuelle (et mathématique) en tant que but à long terme, après avoir spécifié, pensons-nous, la structure causale et la dynamique propre à chaque niveau phénoménal, dans son autonomie conceptuelle et expérimentale et après avoir établi des passerelles conceptuelles qui relient une intelligibilité à une autre, sans nécessairement, ou sans immédiatement, la réduire l'une à l'autre.

\section{Références}

(Preliminary or revised versions of Longo's papers are downloadable from http://www.di.ens.fr/users/longo ). 
Aubin, J.P. Viability theory, 1991, Birkhauser.

Ameisen J.C., La sculpture du vivant. Le suicide cellulaire ou la mort créatrice. Paris, Seuil 1999.

Bailly F., "L'anneau des disciplines", Rev. Int. Syst., 5, n³, 1991.

Bailly F., F. Gaill et R. Mosseri, "La fractalité en biologie : ses relations avec les notions de fonction et d'organisation", in : Biologie théorique (Y. Bouligand Ed.), Ed. CNRS, 1989.

Bailly .F, F. Gaill et R. Mosseri, "Orgons and Biolons" in Theoretical Biology: phenomenological analysis and quantum analogies, Acta Biotheor., 41, p.3, 17, 1993.

Bailly F., Longo G. "Objective and Epistemic Complexity in Biology". Invited lecture, Proceedings of the International Conference on Theoretical Neurobiology, (N. D. Singh, ed.), National Brain Research Centre, New Delhi, INDIA, pp. 62 - 79, 2003.

Bailly F., Longo G. "Space, time and cognition. From The Standpoint of Mathematics and Natural Science". In Causality and Mind, (Peruzzi ed.), Benjamins, Amsterdam, pp. 149-199, 2004.

Bailly F., Longo G. Mathématiques et sciences de la nature. La singularité physique du vivant. Hermann, Paris, 2006.

Bailly F., Longo G. "Schèmes géométriques pour le temps biologique", in preparation, 2006.

Bak P., C. Tang C. and Wiesenfeld K. "Self-organized criticality". Physical Review A 38: 364--374, 1988.

Bernard-Weil E., Stratégies paradoxales en bio-médecine et sciences humaines, Paris, L'Harmattan 2002.

Bourgine P., Stewart J. “Autopoïesis and Cognition”, Artificial Life, 10,3, 2004.

Edelman G., Tononi G. A Universe of Consciousness. How Matter Becomes Immagination, Basic Books, 2000.

Goodwin B.C., Kauffman S.A., Murray J.D., "Is Morphogenesis inherently robust?", J. Theoret. Bio., 1993.

Gould S.J., L'éventail du vivant, Paris, Seuil, 1997.

Horsfield K., "Morphology of branching trees related to entropy", Respir. Physiol., 29, p.179, 1977.

Kauffman S.A., The origins of Order, Oxford U. P., 1993.

Kauffman S., At home in the Universe, Oxford UP, 1995.

Jean R. V. Phyllotaxis : a systemic study in plant morphogenesis, Cambridge University Press, 1994

Lecointre G., Le Guyader H., Classification phylogénétique du vivant, Paris, Belin 2001

Lefèvre J., "Teleonomical optimization of a fractal model of the pulmonary arterial bed", Journ. Theor. Biol., 102, p.225, 1983.

Laguës M., Lesne A. Invariance d'échelle, Belin, Paris, 2003.

Lesne A. Approches multi-échelles en physique et en biologie, Habilitation à diriger la recherhce, Univ. Paris VI, 30/6/2003.

Longo G. "Space and Time in the Foundations of Mathematics, or some challenges in the interactions with other sciences". Invited lecture, First American Math. Soc./SMF meeting, Lyon, July, 2001 (version française, Intellectica, 2003/1-2, n 36-37).

Longo G. "Laplace, Turing and the "imitation game" impossible geometry: randomness, determinism and programs in Turing's test". Invited Lecture, Conference on Cognition, Meaning and Complexity, Univ. Roma II, June 2002. (version française, Intellectica, 2002/2, n. 35).

Mandelbrot B., The fractal geometry of nature, New York, W.H. Freeman, 1982.

Maturana H., Mpdozis J., "El origen de las especies por medio de la deriva natural" rvista Chilena de Historia Natural, 73, 261-310, 2000.

Nicolis G., "Dissipative systems", Rev. Prog. Phys., IL, p. 873, 1986.

Nicolis G., Prigogine I, A la rencontre du complexe, PUF, 1992.

Nicolis G., Prigogine I.., Self-organization in non-equilibrium systems, Wiley, New York, 1977.

Rosen R., Life itself!: A comprehensive Enquire into the Nature, Origin and Fabrication of Life, New York, Columbia University Press, 1991.

Solé R., Goodwin B., Signs of Life, Basic Books, 2000.

Soto A., Sonnenschein C., "The somatic mutation theory of cancer: growing problems with this paradigm?" BioEssays, 26 :1097-1107, 2004. 
Stewart J. "La modélisation en biologie", in Enquête sur le concept de modèle, (Nouvel P., ed.), Presses Universitaire de France, 2002.

Varela F. J.; Maturana, H. R.; \& Uribe, R. "Autopoiesis: the organization of living systems, its characterization and a model". Biosystems 5 187-196, 1974.

Varela F., Autonomie et connaissance, Seuil, Paris, 1989.

Varela F. "The Specious Present: A Neurophenomenology of Time Consciousness", in : Naturalizing Phenomenology (J. Petitot, F. Varela, B. Pachoud, J-M. Roy, Eds.) Stanford University Press 1999.

Waddington C.H., "Stabilization of Systems, Chreods and Epigenetic Landscapes", Futures, 9(2), p.139, , 18, 1977.

West G., Brown J., Enquist B., "A general model for the origin of allometric scaling laws in biology", Science, CCLXXVI, p.122, 1997. 